\title{
OBSERVATIONS ON EARLY COMPONENTS OF THE VISUAL EVOKED RESPONSE AND OCCIPITAL RHYTHMS ${ }^{1,2}$
}

\author{
K. A. KoOI AND B. K. BAgChI \\ Laboratory of Electroencephalography, Neuropsychiatric Institute, \\ University of Michigon Medical Center, Ann Arbor, Mich. (U.S.A.)
}

(Accepted for publication: April 30, 1964)

\section{INTRODUCTION}

The brain responds to intense photic stimulation with a series of rapid deflections which, when instrumentally summated, may be classified by virtue of more or less consistent latency ranges, polarities and distributions. The several deflections of the response are present in the majority of normal subjects but tend to vary, particularly in respect to amplitude and, to a lesser extent, latency. These variations may be of importance in evaluating the functional state or integrity of the brain.

A question of immediate relevance to the clinical interpretation of evoked response patterns may be posed: does the character of the resting pattern suggest that a particular type of evoked response may be encountered? If resting and evoked patterns are highly related, extant knowledge of a physiological and clinical nature about resting rhythms might be germane to the interpretations of evoked responses; if not, or only partially related, the possibility that evoked responses are indicators of a different order or orders of cerebral activity would be enhanced. In addition, recognition of deviations of response characteristics relatable to normal variations of resting activity is essential for the evaluation of responses in patients with brain disorders. That late repetitive components of the response (alpha "ringing") and the alpha rhythm may have inherent similarities has been demonstrated by several workers (Brazier 1958; Barlow 1960; Walter 1961) and is not the direct concern of this paper.

\footnotetext{
1 Supported in part by U.S.P.H.S. Grant NB 02560.

2 Portions of this material were presented at the Joint Meeting of the Western EEG Society and the Central Association of Electroencephalographers, Phoenix, Arizona, February 20-22, 1964.
}

The present study, an extension of a limited analysis previously reported (Kooi and Bagchi, in press), evaluates early deflections, i.e., those occurring prior to $250 \mathrm{msec}$, each wave being considered individually. Amplitude-latency relationships for each response component are also examined. A related study is that of Ebe $e t$ al. (1962) who identified four types of evoked responses in normal subjects. As classified, and on the basis of data gathered by the photographic super-imposition technique, the type of evoked response bore no consistent relationship to resting alpha, beta, theta or delta incidence. Ciganck (1961) concluded similarly that no relationship was ascertainable.

\section{SUDJECTS AND PROCEDURE}

Non-hospitalized, gainfully employed adults were studied. Each subject as also evaluated medically, as part of a continuing intensive periodic health appraisal program (Tupper and Beckett 1958). The health appraisal included a formal ophthalmological examination. Neurolog* ical consultation was requested as indicated. One hundred individuals of the initial 113 studied were free of ophthalmological and neurological disorders. The thirteen were rejected because of various eye disorders. Five subjects with low voltage random frequency records were added to the initial group in order to increase the total number of subjects with this relatively uncommon type of resting pattern. These subjects were selected from a second series of 89 normal individuals similarly studied frnm the medical standpoint. Age range for the entire group of subjects (105) was from 28 to 65 with an average of 47.4. Ninety-nine were males; six were females.

The subjects sat in a comfortable chair with 
the face of a Grass PS-2 photic stimulator lamp immediately before the open eyes. The lamp was enclosed in a double-wall box which eliminated subjective awareness of the lamp "click". The effectiveness of the encasement was evidenced by the fact that no response was detectable when the subject's eyes were shielded from the light. Light diffusion and elimination of internal lamp detail was accomplished by an opal glass face. The subjects were requested to gaze directly into the carefully centered lamp. The rated lamp intensity of 200,000 foot candles was reduced approximately $20 \%$ by the opal glass. Controlled background light intensity was adjusted to be 1 foot candle at the subject's eyes.

A $20 \mathrm{~min}$, eyes-closed, resting EEG tracing was obtained prior to the evoked response study. The evoked responses were summated with the Mnemotron CAT, the 8-channel EEG recording being continued throughout the procedure. The instant of each flash was marked on the EEG record. The summated curves were displayed on a slave Tektronix CRO and photographed with a Hathaway oscilloscope camera taking 6" wide paper. Square wave system calibration was carried out with an ENSCO low frequency function generator. Measurements of average amplitudes were obtained by comparing the summated curves with a $10 \mu \mathrm{V}$ calibration voltage processed in a manner identical to the evoked responses and similarly displayed and photographed.

Data were gathered from four regions simultaneously, using selected infraorbital, central, parietal, occipital and cervical electrodes paired with combined ear reference. The data formally analyzed in the present study were those derived with central-ear and occipital-ear connections. Three series of accumulations were carried out. In the first two, 50 responses were summated with an analysis period of $250 \mathrm{msec}$, the flash being randomized and presented once every 4-6 sec. For the last series, 300 responses were accumulated, with a $1.25 \mathrm{msec}$ analysis period, the light flashing at $0.5-1 \mathrm{sec}$ intervals. The statistical evaluation was based upon the initial $n 50$ accumulation. This series was selected rather than the higher $n$ series because preliminary studies indicated (1) greater response stability with long flash interval technique, possibly functions of less pupii variance and subjective discomfort and (2) amplitude reduction of the major occipital surface negative component at flash rates of $1 / \mathrm{sec}$ or faster. The similarity of each subject's response patterns for successive $n 50$ accumulations without procedural changes and the rarity of appreciable cervical muscle artifact were established earlier (Kooi and Bagchi, in press).

Occipital electrodes were $3 \mathrm{~cm}$ above the inion and $3 \mathrm{~cm}$ from the midline. Central electrodes were $4 \mathrm{~cm}$ from the midline on the interaural plane. All measurements were made from left-sided leads.

Resting eyes-closed EEGs were classified in respect to broad range alpha amplitude, incidence and modulationcriteria.Averagealpha amplitude, the average of recurrent maximum levels during periods of sustained alpha elaboration, was judged to the nearest $10 \mu \mathrm{V}$. These levels, which ranged from 10 to $80 \mu \mathrm{V}$, were grouped for the three alpha amplitude categories: $20 \mu \mathrm{V}$ ani" below; 30 and $40 \mu \mathrm{V}$; and above $40 \mu \mathrm{V}$. Alpha incidence (percent time rhythmic alpha frequency activity, irrespective of amplitude) ranges were $0-20 \%, 21-80 \%$ and $81 \%$ or more. Modulation was judged to be good if it was monorhythmic, sinusoidal and regular in spindling characteristics.It was termed "fair"or" poor" according to degree of moment to moment frequency and amplitude variability. Amplitude, incidence and modulation were evaluated from the initial and final 2 min scalp-ear runs.

Alpha frequency $(8.5-13 \mathrm{c} / \mathrm{sec})$ was determined by counting two $5 \mathrm{sec}$ periods of maximally developed alpha activity during the initial and final 2 min scalp-ear runs and averaging the values. The correlation between the two counts was 0.89 .

A separate alpha persistence index was derived from the EEG recording with eyes open during computation of the averaged evoked response. This measure indicated the number of times that two or more alpha waves exceeding $20 \mu \mathrm{V}$ were present immediately preceding the instant of each of the 50 stimuli. The $20 \mu \mathrm{V}$ level was arbitrarily selected in an attempt to quantify the degree of alpha "blocking" while the test was in progress. Subjects varied widely in this respect with indices ranging from 0-28. Of methodolog-

Electroenceph. clin. Neurophysiol., 1964, 17: 638-643 
ical interest was the fact that the alpha persistence index was highly related to the alpha amplitude of the resting record. Averages of 0.6, 4.1 and 12.0 were obtained for the three alpha amplitude groups (as defined above), ordered from low to high. These means were significantly different from one another beyond the 0.001 level of confidence.

Peak latencies and amplitudes were measured for all major waves of the occipital (hereinafter designated " $O$ ") evoked response prior to 250 msec. Latency ranges for these waves, systematically determined in 100 subjects earlier, were limited to: wave OI (surface neg.) - 35-60 msec; OII (pos.) - 55-90 msec; OIII (neg.) - 80-110 msec; OIV (pos.) - 100-140 msec; OV (neg.) 130-160 msec. Later waves were sufficiently inconstant so that they could not be statistically evaluated. If two major waves of the same polarity fell within a pre-established latency range, the greater amplitude wave was measured and tabulated. The surface negative vertex sharp wave (CV), maximal over central regions with latencies between 110 and $160 \mathrm{msec}$ was also measured. Amplitudes were trough-peak or peak-trough, e.g., amplitude of OII was the overall deflection from the peak of $O I$ to the trough of Oll (Table I).

The significunce of differences between means for the various EEG categories was evaluated by 1 tests. Pearson product-moment correlation coefficients were computed when uppropriate. Sixty individual $t$ tests and $\mathbf{4 5}$ product-moment correlation coefficients were carried out.

\section{RESULTS}

Inspection of individual response curves of subjects falling within each of the resting EEG classifications revealed no major differences in their patterns. Instances of well developed or absent subcomponents were observed in each EEG category. The statistical analysis revealed, however, significant low order relationships in respect to certain aspects of the response and the spontaneous pattern.

Resting alpha amplitudes of 21 subjects were $20 \mu \mathrm{V}$ or below. Fifty-two subjects had alpha amplitudes of 30 and $40 \mu \mathrm{V}$. In the remaining 32 subjects alpha amplitude exceeded $40 \mu \mathrm{V}$. Average amplitudes of response subcomponents
TABLE I

Visual evoked response.

Average amplitudes of individual components of $\mathbf{5 0}$ averaged responses $v s$. alpha amplitude (microvolts)

\begin{tabular}{lccc}
\hline & $(1)$ & $(2)$ & $(3)$ \\
& $<20$ & $20-40$ & $>40$ \\
\hline OI & $4.0(21)^{1}$ & $3.6(52)$ & $3.2(32)$ \\
OII & $6.6(20)$ & $6.5(49)$ & $8.1(31)$ \\
OIII & $7.8^{2}(21)$ & $9.8^{2}(51)$ & $13.7^{2}(31)$ \\
OIV & $7.3^{2}(19)$ & $9.9(45)$ & $11.8^{2}(27)$ \\
OV & $7.3(18)$ & $8.5(45)$ & $7.3(27)$ \\
CV & $13.7(21)$ & $13.5(52)$ & $14.2(30)$ \\
\hline
\end{tabular}

1 Numbers in parentheses indicate subjects included in average.

2 OIII-(1) vs. (3) $p<0.01$, (2) vs. (3) $p<0.05$ OIV-(1) vs. (3) $p<0.02$.

for the three alpha amplitude groups are presented in Table 1. Amplitudes of OIII and OIV tended to be greater in individuals with higher resting alpha voltages. No significant differences were found for OI, OII or OV in this respect. It is of interest that the amplitude order of OI was opposite that of OIII and OIV (see Table I, OI vs. OIII and OIV). OI is of additional interest in relation to resting alpha amplitude inasmuch as its peak latency tended to be longer in those subjects with low amplitude patterns; average latencies were $43.5 \mathrm{msec}, 40.5 \mathrm{msec}$ and $\mathbf{3 8 . 0}$ msec for the three ascending alpha amplitude groups. The first and second mean latencies were significantly different at the 0.05 level of confidence; the first and third were beyond the 0.01 level. Latencies of other deflections did not vary in relation to alpha amplitude.

Amplitudes and latencies of the vertex sharp wave (CV) were also compared for the three alpha groups. Both varied widely in each group with similar mean values. No significant differences were found.

Alpha incidence groupings - low, moderate and high - roughly paralleled those for alpha amplitude and the findings were in the same direction. However, no significant relationships between resting and response characteristics emerged except that for latency of OI. Low alpha incidence subjects had longer OI latencies than those with high $(t=2.6, p=0.01)$. Average latency for the modeiate incidence group fell in between. Average latencies for the three alpha incidence groups were $43.5 \mathrm{msec}, 41.0 \mathrm{msec}$ and

Electroenceph. clin. Neurophysiol., 1964, 17: 638-643 
TABLE II

Visual evoked response.

Latencies and amplitudes vs. alpha index (eyes-open)

\begin{tabular}{lcccc} 
& \multicolumn{2}{c}{ Latency } & \multicolumn{2}{c}{ Amplitude } \\
& $\boldsymbol{r}^{1}$ & Significance & $\boldsymbol{r}^{1}$ & Significance \\
\hline OI & -0.25 & 0.01 & -0.13 & - \\
OII & -0.23 & 0.02 & +0.13 & - \\
OIII & -0.03 & - & +0.19 & - \\
OIV & +0.08 & - & +0.05 & - \\
OV & +0.25 & $<0.02$ & -0.18 & -
\end{tabular}

1 In this and subsequent tables, $r$ is the Pearson product-moment coefficient of correlation.

\section{0 msec.}

The alpha persistence index, a measure of the degree of alpha blocking during accumulation of the summated response, related significantly to the latencies of OI, OII and OV (Table II). OI and OII latencies tended to be longer in association with a low persistence index whereas OV appeared earlier. Amplitudes of the several components studied did not vary consistently in relation to this index.

Modulation of the resting alpha rhythm was good in 61 subjects, fair or poor in 28. Sixteen subjects were eliminated because they had no sustained alpha activity. The evoked responses were not appreciably different for the two groups.

TABLE III

Visual ovoked response. Latencies ts. alpha frequency

\begin{tabular}{lcc} 
& $r$ & Significunce \\
OI & -0.03 & \\
OII & -0.02 & $\cdots$ \\
OIII & +0.01 & - \\
OIV & -0.14 &.- \\
OV & -0.27 & 0.02 \\
CV & -0.16 & - \\
\hline
\end{tabular}

${ }^{1}$ See Table II

Alpha frequency was examined in relation to latencies of OL through OV (Table III). Twentyone subjects were excluded from analysis on the basis of lack of sustained alpha activity and/or poor alpha modulation. These variables appeared to be unrelated except for wave $V$. This component tended to have a shorter latency in those subjects with faster alpha rhythms.

Preliminary inspection of the averaged response waves led to an impression that greater response amplitudes were regularly associated with longer peak latencies. Statistical evaluation corroborated this impression for all waves except OV (Table IV).

TABLE IV

Visual evoked response.

Amplitude vs. latency of individual waves

\begin{tabular}{lcc}
\hline & $\boldsymbol{r}^{1}$ & Significance \\
\hline OI & +0.38 & $<0.01$ \\
OII & +0.23 & 0.02 \\
OIII & +0.51 & $<0.01$ \\
OIV & +0.32 & $<0.01$ \\
OV & -0.01 & - \\
CV & +0.29 & $<0.01$ \\
\hline
\end{tabular}

1 See Table II

Pupil size, in resting conditions, ranged from 2.5 to $7.0 \mathrm{~mm}$ with an average of $4.5 \mathrm{~mm}$. It was not found to be a significant factor in determining response latencies or amplitudes, nor was it related to resting alpha amplitude. The correlation between pupil measurements, taken immediately before and after recording of evoked responses, was 0.94 . Correlations between pupil size and parameters of individual waves were all near zero. Latency of $O I$ was further examined in relation to pupil size by restricting the correlation to those subjects who had OI latencies falling within 34 to $45 \mathrm{msec}$, the range of must frequent peak culmination. No improvement of the correlation emerged.

\section{DISCUSSION}

The present study was undertaken to ascertain whether characteristics of an individual's resting occipital pattern, as routinely assessed in the clinical laboratory, offer a basis for anticipating certain types of variation of the form of the visual evoked response. The findings suggest that several types of interislationships may be significant. The low orders of the relationships disclosed are not unexpected inasmuch as (1) surface electrical records are inevitably indistinct representations of the primary events, (2) the visual approach to analysis of spontaneous activity limits degree of mathematical quantification possible and (3) the final form of the evoked response may be largely the resultant of anatomical and physiological factors which are unrelated to the resting electroencephalogram. Nonethe- 
less, the endeavor is of some pragmatic value and, with attention to levels of significance encountered, of theoretical interest.

If it is accepted that latencies and amplitudes of visual evoked responses may be affected in cisorders of the brain, estimation of the extent of alteration requires awareness of factors that might "normally" be associated with variations of these wave parameters. Thus, in this frame of reference, our findings indicate that the individual with a low voltage record tends to have late culmination of OI. Amplitudes of OIII and OIV may be slightly greater than average in the subject with a high amplitude alpha rhythm. $O V$ is more likely to culminate in the early portion of its general range if the subject has a fast alpha frequency. These findings, taken in conjunction with the demonstrated relationships between amplitudes and latencies of individual subcomponents - OI, OII, OIII, OIV and CV - are useful in identifying normal variant responses.

The divergence in behavior of the amplitude and latency of OI from those of OIII and OIV suggests that they may be the results of functionally distinet processes. It may also be argued that $O V$ is yet another functionally different wave. It is the only wave whose latency reflects the alpha frequency and is positively related to the alpha persistence index. In addition, its amplitude does not follow the trend of OII, OIII and OIV, nor are its latency and amplitude interrelated as for earlier waves. CV, readily distinguishable from other events on the basis of characteristic distribution, latency and functional lability, is yet another separable component. However, and rotwithstanding these evidences of differences between several components of the response in man, until their functional roles have been clearly delineated, analogies to primary and secondary responses as described in animals (Derbyshire et al. 1936; Forbes and Morison 1939; Brazier 1954; Buser and Borenstein 1957; Tories and Warner 1962) remain hypothetical.

$O V$, a surface negative component which has a latency range of 130-160 msec, emerges as a wave of special interest in relation to the alpha mechanism. Its timing, in terms of moment of appearance, latency range and correspondence with alpha frequency, makes it likely that this component is linked to cerebral processes underlying the alpha rhythm. That a wave with alpha-like characteristics should be present as a component of the visual evoked response in man is not unexpected in view of the parellelism noted by Barlow (1960) between frequencies of resting alpha activity and after-discharge to a photic stimulus. Attention is drai $n$ here particularly to the point at which the phenomenon is first detectable and its initial form.

The positive correlation of the latency of $\mathrm{OV}$ with the alpha persistence index is a challenging observation inasmuch as it discloses a second relationship that may influence the exact timing of OV. The lengthening of its latency which may be associated with high alpha persistence during collection of responses might operate to obscure or exaggerate the alpha frequency - OV latency relationship. Further studies will be necessary to elucidate the precise nature and meaning of these and other relationships suggested by this exploratory analysis.

\section{SUMMARY}

One hundred and five adults, free of neurological or ophthalmological disorder, were studied in order to assess possible interrelationships between their resting EEG patterns and waves I through $V$ of the occipital evoked response and the vertex sharp wave. The initial (35-60 msec) occipital surface negative wave tended to have a longer peak latency in those individuals with (1) low voltage resting records and (2) low alpha persistence during response summation. Am, plitude variation of occipital waves III and IVsurface negative and positive respectively, tended to parallel that of the resting alpha rhythm. The latency of the third occiput negative component (OV) was inversely related to resting alpha frequency. Longer latencies corresponded with higher amplitudes for occipital waves $I$ through IV and the vertex sharp wave. The vertex sharp wave did not vary in relation to resting alpha amplitude, incidence or frequency.

The authors wish to recognize the important contributions of W. B. Wickland, M. D. Drescher and W. F. Kohl for technical assistance and J. Candy in data processing. Appreciation is also extended to Miss Ester Schaeffer of the University of Michigan Statistical Research Laboratory for her willingness to compute the large number of statistical evaluations that the study design required. 


\section{REFERENCES}

Barlow, J. S. Rhythmic activity induced by photic stimulation in relation to intrinsic alpha activity of the brain in man. Electroenceph. clin. Neurophysiol., 1960, 12: 317-325.

Brazier, M. A. B. The action of anesthetics on the nervous system with special reference to the brain stem reticular formation. In J. F. Delafresnay (Ed.), Brain mechanisms and consciousness. Thomas, Springfield, Ill., 1954: 163-199.

Brazier, M. A. B. Studies of evoked responses by flash in man and cat. In H. H. JAsper, L. D. Proctor, R. S. Knighton, W. C. Noshay and R. T. Costello (Eds.), Reticular formation of the brain. Little, Brown and Co., Boston, 1958: 151-168.

Buser, P. et Borenstein, P. Réponses corticales "secondaires" a la stimulation sensorielle chez le chat curarisé non anesthèsié. Electroenceph. clin. Neurophysiol., 1957, Suppl. 6: 89-108.

CIGÁNEK, L. Die elektroencephalographische Lichtreizantwort der menschlichen Hirnrinde. Verl. Slowak. Akad. Wissensch., Bratislava, 1961.

Derbyshire, A. J., Rempel, R., Forbes, A. and Lam-
BERT, E. F. Effects of anesthetics on action potentials in the cerebral cortex of the cat. Amer. J. Physiol., 1936, 116: 557-596.

Ebe, M., Mikam, T., Aki, M. and Miyazaki, M. Electrical responses evoked by photic stimulation in human cerebral cortex. Tohoku J. exp. Med., 1962, 77: 352-366.

Forbes, A. and Morison, B. R. Cortical response to sensory stimulation under deep barbiturate narcosis. J. Neurophysiol., 1939, 2: 112-128.

KoOI, K. A. and BAGCHI, B. K. Visual evoked responses in man: Normative data. In R. KATzMAN (Ed.), Sensory evoked responses in man. Ann. N. Y. Acad. Sci., in press.

Torres, F. and WARner, J. S. Some characteristics of delayed responses to photic stimuli in the cat. Electroenceph. clin. Neurophysiol., 1962, J4: 654-663.

Tupper, C. J. and Beckert. M. B. Faculty health appraisal, University of Michigan. Univ. Mich. med. Bull., 1958, 24 : 35-43.

Walter, W. G. "Summary". In M. A. B. Brazier (Ed.), Compurer techniques in EEG analysis. Electroenceph. clin. Neurophysiol., 1961, Suppl. 20: 81-87.

Reference: KooI, K. A. and BAGCHI, B. K. Observations on early components of the visual evoked response and occipital thythms. Electroenceph. clin. Neurophysiol., 1964, 17: 638-643. 